\title{
Questes
}

vestes Revue pluridisciplinaire d'études médiévales

$9 \mid 2006$

À la marge

\section{Le sens à la marge}

Le statut des gloses marginales dans le manuscrit de Venise (Marciana, fr. app. 23) des Eschés amoureux

\section{Amandine Mussou}

\section{(2) OpenEdition}

1 Journals

\section{Édition électronique}

URL : http://journals.openedition.org/questes/2036

DOI : 10.4000/questes. 2036

ISSN : 2109-9472

Éditeur

Les Amis de Questes

\section{Édition imprimée}

Date de publication : 15 juin 2006

Pagination : $2-10$

ISSN : 2102-7188

\section{Référence électronique}

Amandine Mussou, «Le sens à la marge », Questes [En ligne], 9 | 2006, mis en ligne le 01 janvier 2014, consulté le 19 avril 2019. URL : http://journals.openedition.org/questes/2036 ; DOI : 10.4000/ questes.2036

\section{(C) Association des amis de «Questes »}




\section{Le sens à la marge}

\section{Le statut des gloses marginales dans le manuscrit de Venise (Marciana, fr. app. 23) des Eschés amoureux}

\section{Amandine MUSSOU}

La postérité des Eschés amoureux en vers, récit allégorique anonyme composé vers 1370-1380, a surtout été assurée par Évrart de Conty, qui en a proposé un commentaire en prose, Le livre des eschez amoureux moralisés. La version en vers joue délibérément avec l'intertexte du Roman de la Rose. Nature apparaît un matin au narrateur et lui reproche de ne pas parcourir le monde. Le récit expose ensuite les pérégrinations du jeune homme, appelé « l'acteur », jusqu'à son arrivée au verger d'Amour. Là, le récit s'éloigne de son modèle puisque l'acteur aperçoit Déduit, le dieu du jeu, affrontant une jeune fille aux échecs. À l'issue de la partie, le dieu d'Amour ordonne au narrateur de prendre la place de Déduit et de jouer contre la demoiselle. Il est maté par celle-ci. À la suite du récit de cette défaite, Pallas, la déesse de l'entendement, délivre au narrateur, dans un long discours encyclopédique et didactique, une série de leçons. La partie narrative ne couvre que le premier tiers des trente mille vers du texte ; la partie discursive occupe les deux tiers restants.

Ce texte est conservé dans deux manuscrits. Celui de Dresde Sächsische Landesbibliothek, Oc. 66 - contient la version la plus longue, bien qu'inachevée; il a été fortement endommagé pendant la Seconde Guerre 
mondiale. Le manuscrit de Venise - Marciana, fr. app. 23 - est fragmentaire, mais la quasi-totalité de la partie narrative et le début du long discours de Pallas y figurent. Il comporte surtout une particularité formelle : la présence de gloses marginales en latin, d'une main différente de celle du copiste, notamment aux moments où le sens allégorique appelle le plus de commentaires.

La partie d'échecs est précédée d'une description minutieuse de l'échiquier et des pièces. Il s'agit d'un objet luxueux qui suscite l'admiration et l'émoi de l'acteur. Sur le modèle de l'échiquier de Jacques de Cessoles, l'écu de chacune des pièces comporte un emblème. La description de l'objet couvre plus de trois feuillets et les gloses latines envahissent alors les marges. Elles explicitent le sens de chacun des emblèmes en établissant une équivalence entre ces derniers et des figures allégoriques. Sur le premier pion de la jeune fille figure ainsi un croissant de lune nouvelle ; le commentaire latin y associe Jeunesse. L'absence de ces gloses dans le manuscrit de Dresde nous conduit à examiner leur statut et leur lien avec le corps du texte. Le sens allégorique qu'elles délivrent est-il intégré au récit ou demeure-t-il à la marge ?

\section{Le travail des marges}

Si l'échiquier est décrit de façon très précise dans le manuscrit de Venise, les pièces ne représentent rien de façon allégorique. L'équivalence entre les images et leur signification n'est posée que par les gloses, à la marge du texte. 
$\mathrm{Au}$ folio $66 \mathrm{r}^{\circ}$ figure un diagramme représentant un échiquier. ${ }^{1} \mathrm{La}$ position des pièces est celle du début de la partie, chacune d'elles étant désignée non par l'emblème décrit dans le texte en vers, mais par l'allégorie établie par le commentaire latin. Après la description des pièces et celle de la partie d'échecs, le procédé d'allégorisation est répété. Ce diagramme, tracé par la même main que les gloses, les illustre bien plus qu'il ne dialogue avec le texte lui-même. Sa place a été prévue dans le manuscrit puisqu'il occupe toute la largeur de la page et clôt l'épisode consacré à la partie d'échecs. L'un des problèmes soulevés par le roman allégorique réside dans le rapport du texte à la glose : où et comment expliquer le sens parfois obscur d'un symbole, d'un emblème ou d'une allégorie ? Le manuscrit de Venise semble choisir une solution extrême puisque s'opère une séparation physique des deux éléments : le texte en vers d'une part et la glose latine en prose, reprise par le graphique, d'autre part. Le bilinguisme accentue cette distinction. Le sens allégorique est donné en prose latine, dans la langue propre à l'expression du vrai, quand la matière brute est livrée en vers français. Sur le diagramme, les allégories sont toutefois écrites en français. Les marges elles-mêmes font un usage double de ce bilinguisme. Les explicitations allégoriques en latin sont souvent relativement longues et se décalent par rapport aux vers qu'elles commentent. Sur l'autre marge, des indications en français rappellent, face au vers décrivant l'emblème, l'allégorie correspondante.

Selon Gianmario Raimondi², le manuscrit de Venise serait la copie la plus fiable des deux manuscrits. Il affirme même qu'il s'agit sinon de

\footnotetext{
${ }^{1}$ Voir ci-contre.

2 Gianmario RAIMONDI, Les Eschés amoureux. Studio preparatorio ed edizione, in Pluteus, 8-9, 1990-1998, Alessandria, Edizioni dell'Orso, p. 67-241. 
A la marge

Questes, $\mathrm{n}^{\circ} 9$ 
l'original, du moins d'un manuscrit de travail, derrière lequel transparaît une volonté d'auteur - tout un travail de réécriture est en effet à l'œuvre. Les commentaires marginaux sont l'une des manifestations de cette vision globale. Les gloses font partie d'un système de travail sur le texte, qui ne se contente pas d'apporter des précisions ou des références. Ces commentaires donnent un sens à l'œuvre - notamment à ce passage - et s'intègrent dans un système interprétatif relevant d'un projet auctorial.

\section{Gloser à la marge}

Le commentateur explicite le sens des emblèmes figurant sur les écus des figures. Cette voie au commentaire avait été ouverte dès le début de l'épisode :

« Mais encor plus m'esmerveilloient

De che que je vi qu'il avoient

Aussy com pour descongnissanche

Ou pour aultre seignifianche,

Trestuit fourmes et ymaigettes

Par dedens lors escus pourtrettes... $»^{3}$

Il s'agit donc d'éclaircir cette « senefiance ». La valeur explicative des marges est mise en évidence par les formules utilisées: "significatur », « intelligetur », « designatur »... Un adverbe vient régulièrement souligner la pertinence du choix - « significatur convenientissime », « optime ». La glose est souvent construite en trois temps : l'évocation de quelques traits marquants de l'emblème, une formule de comparaison - « et ita », « similiter » - et le passage à l'allégorisation. Le septième des huit pions de la jeune fille

${ }^{3}$ Christine KRAFT, Liebesgarten-Allegorie der Echecs amoureux, Kritische Ausgabe und Kommentar, Frankfurt am Main, Peter Lang, 1977, p. 119. 
comporte ainsi sur son écu une panthère, qui représente Bonté. Cet animal est caractérisé par la variété des couleurs de sa robe et son odeur suave, affirme la glose. De la même façon, la jeune fille vertueuse est ornée d'une série de qualités et sa bonne réputation émet un doux parfum. ${ }^{4}$

Les mécanismes qui permettent le passage de l'emblème à l'allégorie reposent sur l'analogie. Celle-ci est de différents types. L'un des fous du narrateur porte une nef et la figure allégorique qui lui est associée est Espérance. La glose mêle deux types d'arguments pour opérer ce rapprochement : une analogie thématique - le navire amène les voyageurs à bon port, comme l'espérance guide l'amant - et formelle - le navire est étroit en son début et en sa fin et large au milieu, de même l'espoir de l'amant varie selon une courbe ascendante puis descendante. ${ }^{5}$

Les gloses ont bien souvent une dimension prescriptive : elles exposent la façon dont l'être aimé et l'amant doivent se comporter, délivrant à la marge du texte un véritable art d'aimer. L'un des pions du narrateur porte un cygne qui représente Beau Maintien. Le cygne a des plumes blanches et une chair noire, est-il dit en latin; il chante au moment de mourir. De même, l'amant doit feindre de ne pas aimer la jeune fille et resplendir de toutes ses vertus, mais brûler d'amour intérieurement. Ce n'est qu'en dernier lieu qu'il peut révéler son amour. ${ }^{6}$ Les gloses posent un lien de type métonymique entre les

\footnotetext{
${ }^{4}$ Ibid., p. 236 : «Septimus est Bontés, que per pantheram significatur, cuius cutis multis coloribus est distincta et odor suavissimus ; (...) et ita mulier virtuosa et bona multis bonis conditionibus decoratur. Odorem suavissimum emittit per bonam famam et sic de aliis. »

${ }^{5}$ Ibid., p. 241 : «Duo alphini sunt Espoir et Desir. Primus per navem significatur, que peregrinos portat ad portum salutis. Item navis est stricta in prinicipio et in fine et lata in medio. "

${ }^{6}$ Ibid., p. 240 : «Septimus est Biau Maintien, qui per cygnum propriissime intelligetur, quia in aquis habitat, quia mature et ordinate incedit, quia fortis est alis; habet etiam plumas albas exterius et carnes nigras interius et in morte canit. Ita similiter amans prudens debet habere providentiam, maturum et gratum incessum, exterius fingere non amare et
} 
pièces de l'échiquier et le joueur qui les déplace. Toute la métaphore des échecs dans le récit repose sur ce rapport puisqu'en perdant au jeu, le narrateur perd évidemment son cœur. Les rois des deux joueurs n'ont pas les mêmes emblèmes, mais leurs allégories se correspondent. Il s'agit de Franche Volonté - appelée également Cœur pour la jeune fille et Frans Voloirs pour l'acteur. Le graphique du folio $66 \mathrm{r}^{\circ}$ souligne cette symétrie : «li drois rois des eschés c'est li coers amoureux ou li coraiges ${ }^{7}{ }^{7}$ est-il écrit sur les deux cases où figurent ces rois. C'est le roi qu'il faut prendre aux échecs, comme c'est le cœur qu'il faut prendre en amour. La pièce maîtresse du jeu trouve ainsi un équivalent amoureux de taille.

Le personnel allégorique du Roman de la Rose est convoqué à la marge dans ce passage. Si le lien intertextuel est présent dans la narration des Eschés amoureux, il s'efface lors de la partie d'échecs, qui est une création originale. Les gloses semblent le rétablir. Oiseuse, qui ouvre la voie à l'amant dans le Roman de la Rose, est représentée par le premier pion du narrateur. Certains des membres de l'armée d'Amour sont également présents dans les deux camps - Noblesse, Franchise, Pitié, Simplesse, Beauté, Jeunesse pour la jeune fille et Hardement, Delis, Pacience, Bien Celer pour le narrateur. Cette intertextualité conserve les structures du modèle puisque deux couples d'allégories se retrouvent dans le jeu d'échecs, qui permet le maintien de cette construction binaire : les deux fous de la jeune fille sont Franchise et Pitié, qui interviennent chez Guillaume de Lorris pour neutraliser l'opposition de Danger, et ses deux cavaliers représentent Honte et Peur, deux compagnons du même Danger. Les gloses délivrent à la marge du texte un art d'aimer

virtutibus resplendere, sed interius debet esse amore totus ustus, et in fine debet statum suum amice sue revelare. "»

${ }^{7}$ Voir p. 5 de ce numéro. 
convoquant un personnel allégorique largement inspiré du Roman de la Rose, au moment où ce modèle semble oublié.

\section{$\underline{\text { 3. De la marge au centre }}$}

La confrontation des Eschés amoureux avec leur réécriture en prose est riche d'enseignements quant au statut des gloses marginales. Les analyses de Gianmario Raimondi prouvent qu'Évrart de Conty a certainement connu une version proche de celle que lègue le manuscrit de Venise, puisqu'une variante qui n'apparaît pas dans le manuscrit de Dresde est convoquée dans le texte en prose. ${ }^{8}$ L'étude des gloses semble confirmer cette filiation. En effet, l'œuvre d'Évrart de Conty développe largement les interprétations allégoriques des emblèmes : la description des pièces et l'explicitation des figures dessinées sur les écus couvrent par exemple quarante-trois feuillets dans le manuscrit BNF, fr. 24295, du Livre des eschez amoureux moralisés. Les équivalences allégoriques sont les mêmes que celles qu'établit la glose du manuscrit de Venise. L'amplification de la matière explicative donnée à la marge du texte initial est ici manifeste.

Si le déroulement de la partie est glosé dans le manuscrit de Venise, il l'est moins que la description des pièces qui le précède. La signification d'un coup est parfois commentée. Chez Évrart de Conty, la dénomination des pièces par l'allégorie permet de développer ces commentaires et de rendre explicite ce qui n'était que suggéré dans la version en vers. Pour commenter les premiers coups, le narrateur emploie les termes allégoriques, soulignant l'analogie entre les débuts de la partie et la naissance de l'amour :

\footnotetext{
${ }^{8}$ Gianmario RAIMONDI, op, cit., p. 99, note 54 .
} 
« Se nous considerons ces .VI. traiz premiers, nous trouverons qu'ilz segnefient tres proprement l'entree et la premiere occasion d'amours, car les trais de Beauté de Simplece segnifient les graces et les biens des dames amoureuses qui les amans esmeuvent par dehors de premiere venue (...). Les traiz aussi d'autre part de Regart et de Penser qui les comprendent et avisent, les presentent aprés et les offrent au cuer du regardant, qui leur valeur et leur pris considere $(\ldots) \gg{ }^{9}$

De plus, dans quatre des manuscrits conservant le texte d'Évrart de Conty, un schéma représente l'échiquier du manuscrit de Venise. Les pièces sont désignées par les mêmes allégories et sont disposées sur les mêmes cases.

Évrart de Conty choisit d'intégrer au corps de son texte ce qui demeurait marginal dans la version en vers. Les gloses explicatives sont insérées au cœur de la matière qu'il commente. Certes, sa démarche entière repose sur une volonté d'explicitation : dès le début, l'auteur souligne qu'il a composé cet ouvrage sur le modèle des Eschés amoureux en vers pour expliquer ce qui, dans le poème, pouvait sembler obscur. C'est d'ailleurs pourquoi il a choisi de l'écrire en prose, plus accessible que le vers :

«Ce present livre fut fait (...) come pour declairier aucunes choses que la rime contient qui semblent estre obscures et estranges de prime face. Et pour ce fut il fait et ordené en prose pour ce que prose est plus clere a entendre que n'est rime. $\gg^{10}$

Il retient néanmoins de l'épisode de la partie d'échecs à la fois le texte qu'il commente et les gloses du manuscrit de Venise - ou celles d'un manuscrit très semblable dont nous aurions perdu la trace. Le sens allégorique donné à la marge est ici utilisé comme s'il s'agissait d'une partie intégrante du texte luimême. Le commentaire en prose nivelle la distinction établie par la version en vers et choisit d'intégrer les marges au cœur du récit.

9 Évrart de CONTY, Le livre des eschez amoureux moralisés, édition de Bruno RoY, Françoise GuICHARD-TESSON, Montréal, Ceres, 1993, p. 755-756.

${ }^{10}$ Ibid., p. 2. 
D'après le prolongement des Eschés amoureux, il semblerait donc que ces commentaires à la marge aient été considérés comme faisant partie de l'œuvre et non comme des ajouts gratuits ou relevant d'une pure volonté d'érudition. Le sens allégorique qu'ils apportent et développent a semblé nécessaire à l'auteur de la version en prose. Un glissement de la marge vers le centre s'est ainsi opéré à la faveur de cette réécriture. Il est symptomatique de l'entreprise plus générale du commentaire, comme le soulignent les éditeurs du texte d'Évrart de Conty:

«Pour situer d'un mot les Eschez amoureux en prose par rapport au poème, il suffit de dire que cette œuvre est le témoin écrit d'une lectio, au sens médiéval, du poème des Eschez amoureux. Le commentaire latin n'étant qu'un aide-mémoire utilisé par le ou les pédagogues ayant pour mission d' 'enseigner' le poème, un de ces maîtres eut l'idée d'en tirer un exposé écrit, bien articulé et amplifié aux dimensions d'un cycle quasi complet d'études. »" ${ }^{11}$

\footnotetext{
${ }^{11}$ Françoise GUICHARD-TESSON et Bruno ROY in Évrart de CONTY, op. cit., introduction, p. LXVI.
} 